\title{
Multiple metal stenting using a double-balloon endoscope for malignant biliary obstruction in a patient with hepaticojejunostomy
}
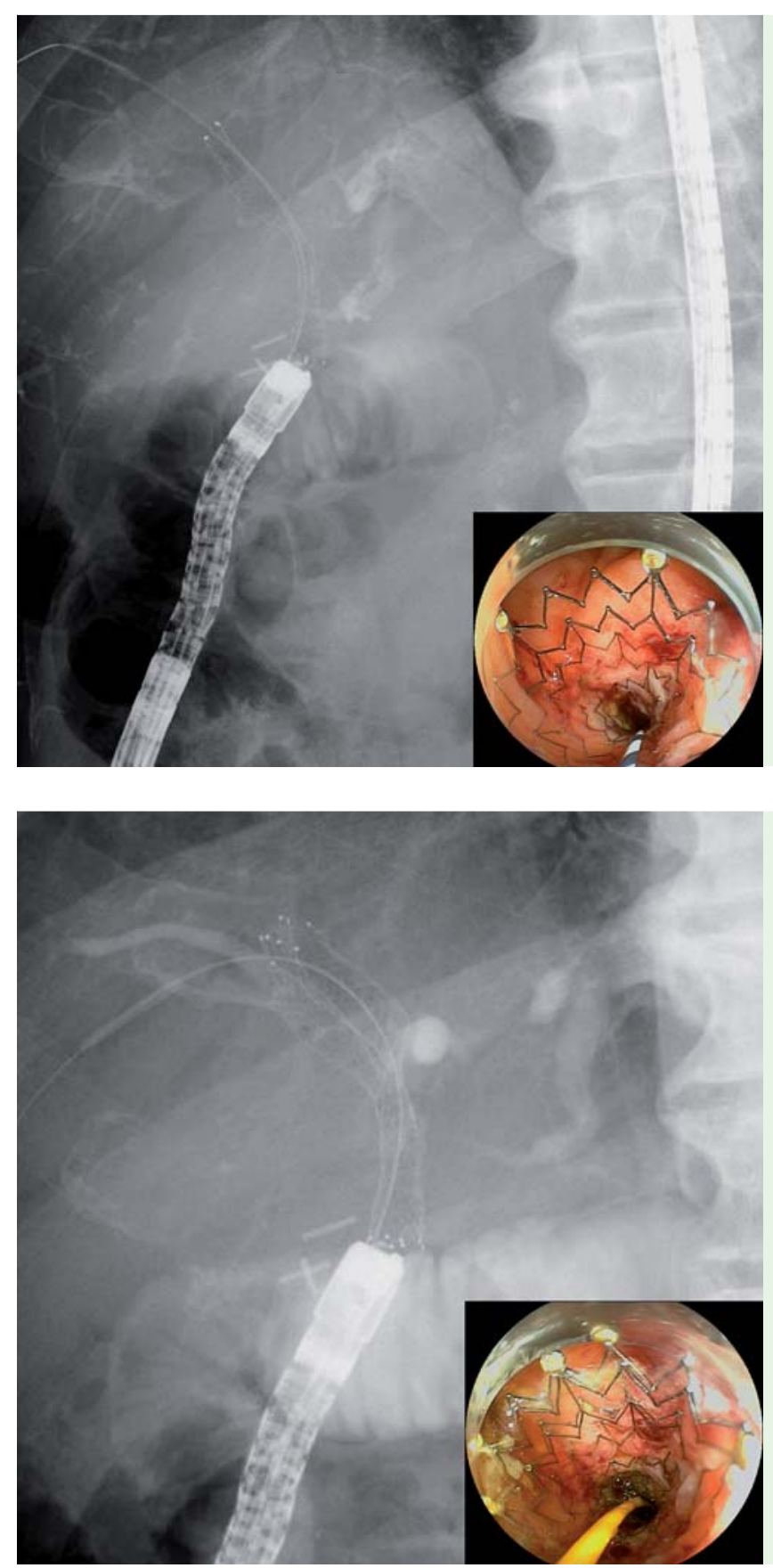

Endoscopic management of malignant biliary obstruction following hepaticojejunostomy can be challenging. With the advent of the short double-balloon endoscope (DBE), therapeutic biliary interventions are possible in surgically altered anatomy [1,2]. Additionally, a novel uncovered metal stent (UMS) has been devel-
Fig. 1 The first uncovered metal stent was deployed into the anterior branch of the right hepatic duct.

Fig. 3 The second uncovered metal stent (UMS) was deployed through the mesh of the initial UMS.

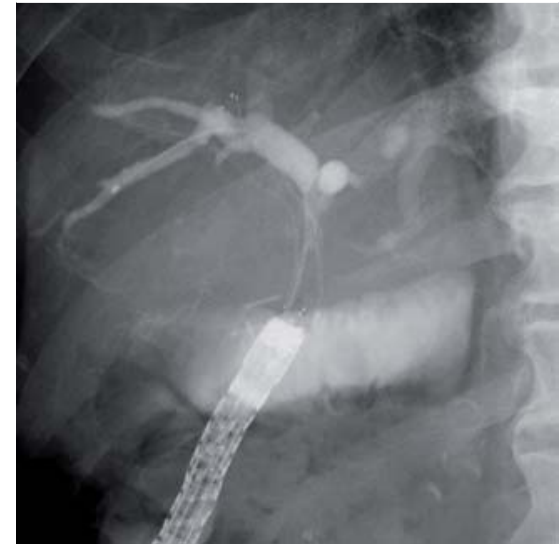

Fig. 2 The catheter was advanced into the posterior branch of the right hepatic duct through the first stent mesh.

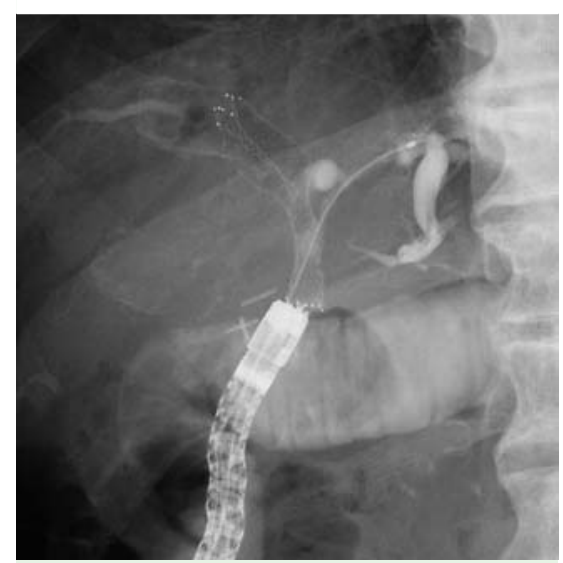

Fig. 4 The catheter was passed into the lateral branch of the left hepatic duct through the overlapping stent mesh.

A 75-year-old man with extrahepatic bile duct cancer underwent pancreaticoduodenectomy with Roux-en-Y reconstruction. He developed cholangitis 11 months postoperatively as a result of liver metastasis in the medial left lobe segment. Endoscopic retrograde cholangiopancreatography (ERCP) was performed to place two plastic stents using a short DBE (EI-530B; Fujifilm Corp., Tokyo, Japan). However, rapid tumor growth resulted in recurrent episodes of cholangitis. Therefore, the decision was made to insert multiple metal stents via another ERCP using the short DBE.

The plastic stents were removed, and the hepaticojejunostomy anastomosis was 


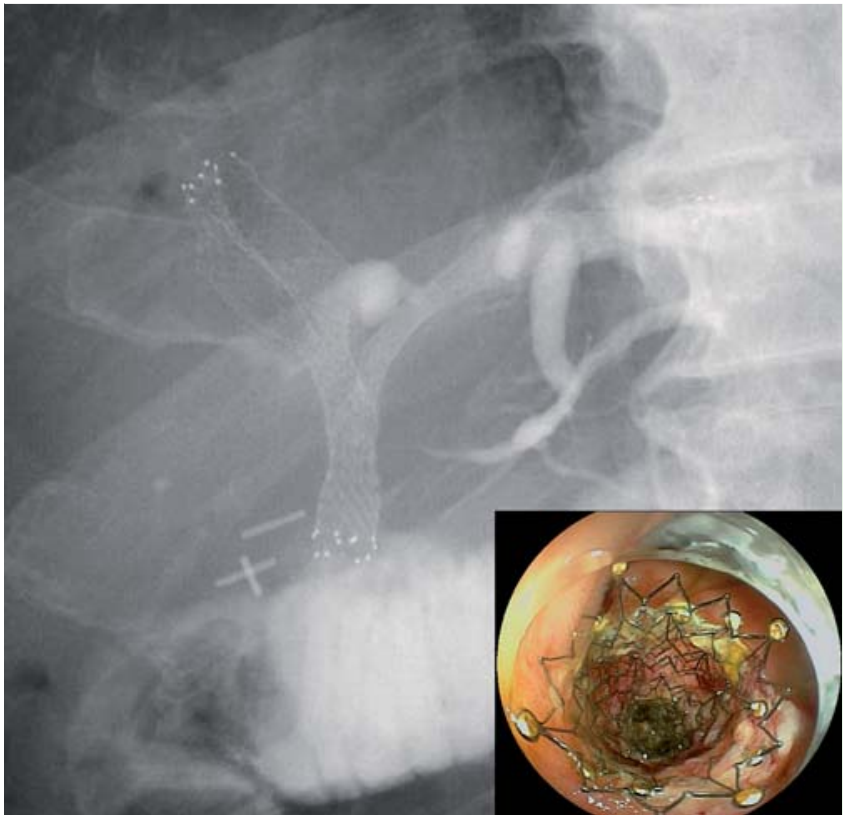

Fig. 5 The third uncovered metal stent (UMS) was deployed through the mesh of the initial and second UMSs.

cannulated. A 0.025-inch stiff guidewire (Revowave; Piolax Medical Devices, Yokohama, Japan) was placed into the anterior right hepatic duct branch, and the UMS was inserted using the 6-Fr delivery system ( $\bullet$ Fig. 1). A seeking guidewire (Radifocus; Terumo, Tokyo, Japan) sought the posterior right hepatic duct branch through the stent mesh, and the catheter was advanced through the stent mesh ( $\bullet$ Fig.2). The guidewire was exchanged for a stiff guidewire. The second UMS was deployed ( Fig.3). Subsequently, a seeking guidewire sought the lateral left hepatic duct branch through the overlapping stent mesh. The catheter could then be passed through the overlapping stent mesh ( $\bullet$ Fig.4). The guidewire was exchanged. Finally, the third UMS was smoothly deployed into the lateral left hepatic duct using a stent-in-stent method ( $\bullet$ Fig.5).

Endoscopy_UCTN_Code_TTT_1AR_2AZ

Competing interests: None

\section{Hirofumi Kogure, Atsuo Yamada, Hiroyuki Isayama, Tomotaka Saito, Tsuyoshi Hamada, Takashi Sasaki, Natsuyo Yamamoto, Yousuke Nakai, Kenji Hirano, Minoru Tada, Kazuhiko Koike}

Department of Gastroenterology, Graduate School of Medicine, University of Tokyo, Tokyo, Japan

\section{References}

1 Shimatani M, Matsushita M, Takaoka M et al. Effective "short" double-balloon enteroscope for diagnostic and therapeutic ERCP in patients with altered gastrointestinal anatomy: a large case series. Endoscopy 2009; 41: 849-854

2 Tsujino T, Yamada A, Isayama $H$ et al. Experiences of biliary interventions using short double-balloon enteroscopy in patients with Roux-en-Y anastomosis or hepaticojejunostomy. Dig Endosc 2010; 22: 211-216

3 Chennat J, Waxman I. Initial performance profile of a new $6 \mathrm{~F}$ self-expanding metal stent for palliation of malignant hilar biliary obstruction. Gastrointest Endosc 2010; 72 : 632-636

\section{Bibliography}

Dol http://dx.doi.org/

10.1055/s-0034-1377541

Endoscopy 2014; 46: E472-E473

(c) Georg Thieme Verlag KG

Stuttgart · New York

ISSN 0013-726X

Corresponding author Hirofumi Kogure, MD Department of Gastroenterology Graduate School of Medicine University of Tokyo

7-3-1 Hongo, Bunkyo-ku Tokyo 113-8655 Japan

Fax: +81-3-38140021

kogureh-tky@umin.ac.jp 